\title{
L'IDENTIFICAZIONE DI PARTITO IN ITALIA: DUE INDICI A CONFRONTO
}

\author{
di Stefano Palma
}

\section{Premessa}

Componente importante e spesso frequentata degli studi sul comportamento politico, la party identification è un concetto che in Italia e, più in generale, in Europa, è stato sovente utilizzato senza una chiara indagine dei significati che può venire ad assumere in contesti di volta in volta differenti. Sviluppato negli anni '50 dai ricercatori del Michigan Group ${ }^{1}$ come elemento centrale nella spiegazione del comportamento elettorale e delle motivazioni di voto dell'elettore americano, la party identification è stata impiegata svariate volte in contesti nazionali diversi da quello americano per storia, struttura politica e sociale, cultura. Un concetto, dunque, che ha avuto molte «traduzioni», linguistiche e culturali, non sempre ininfluenti sui significati e le dimensioni originarie.

Il modello dell'équipe di Campbell costituisce, tuttavia, un punto di riferimento obbligato, ogniqualvolta si voglia parlare di identificazione di partito; si tratterà poi di stabilire se la nozione di identificazione a cui si fa riferimento possa ancora essere considerata conforme con l'originale costrutto, oppure ci si trovi di fronte ad un nuovo concetto, differente e non riconducibile al primo.

Desidero ringraziare Renato Mannbeimer e gli anonimi referees della «Rivista italiana di scienza politica» per $i$ preziosi suggerimenti.

${ }^{1}$ Il concetto di party identification, dopo la prima presentazione apparsa in Belknap e Campbell (1952), è stato più ampiamente utilizzato nelle successive analisi del Michigan Group: Campbell et al. (1954; 1960; 1966). Un'esposizione analitica degli assunti del modello è nell'introduzione a Budge et al. (1976, 12-13). Relativamente al ruolo assunto dalla party identification all'interno della letteratura politologica, cfr. Prewitt e Nie (1971). 
Perché, infatti, l'identificazione di partito possa essere considerata un utile strumento di analisi, è necessario che le sue caratteristiche fondamentali siano sempre riconoscibili qualora se ne vari la collocazione spaziale o temporale. Al di là dei vantaggi che omogenee rilevazioni di un concetto offrono nelle analisi comparate, è la stessa esistenza dell'identificazione di partito ad essere posta in dubbio ogni volta che mutano i suoi contenuti: «l'identificazione di partito può non esistere al di fuori dell'America, ma non si può cambiarne il significato per forzare la sua esistenza»² (Robertson 1976a, 368).

Il problema diviene ben evidente quando dalla generale definizione del concetto astratto si passa alla sua traduzione in un indicatore che possa venire utilizzato nelle indagini empiriche. L'esigenza di rimanere quanto più possibile fedeli al costrutto teorico originario si scontra, qui, con la necessità di prendere in considerazione lo specifico contesto culturale e sociale implicato nella ricerca, contesto che provoca notevoli dissonanze tra i significati che vengono attribuiti alla domanda dall'intervistato, da un lato, e dal ricercatore, dall'altro.

Così, la traduzione letterale di items da una lingua all'altra, non è affatto un'operazione che preserva il ricercatore dall'inserire elementi di arbitrarietà nella ricerca, in quanto al contrario non offre alcun controllo sui significati realmente implicati. Allo stesso modo, la riproposizione di una identica definizione operativa di un concetto in qualsiasi situazione non garantisce affatto la costanza della sua identità. «La definizione operativa - quindi - deve essere adattata alla situazione spazio-temporale in cui si fa ricerca» (Marradi 1980, 102), operazione di ridefinizione degli indicatori, che, se pur necessaria, apre comunque ulteriori spazi di ambiguità nella ricerca: «infatti, chi può stabilire con certezza se e quanto cambia il concetto, allorché la sua definizione operativa muta per adattarsi alla situazione?» (Marradi 1980,102 ).

Occorre dunque domandarsi quali siano le reali dimensioni lungo le quali l'identificazione di partito si configura - o, meglio, si configurava - nel contesto italiano, le logiche di voto e di agire politico implicate, la qualità del rapporto elettore-partito da essa determinata, l'identità dell'elettore identificato.

2 «Party identification may not exist outside America, but it cannot have its meaning changed to force its existence» (nostra traduzione). 
Non ripercorreremo ora i singoli aspetti del modello del Michigan Group, limitandoci, invece, ad inquadrarne semplicemente i termini generali.

Occorre innanzi tutto ricordare che la party identification definisce un modello di rapporto con il partito politico di natura niente affatto razionale. Si tratta, infatti, di un sentimento di attaccamento personale che viene prodotto dal processo di socializzazione ${ }^{3}$ all'interno della famiglia negli anni della giovinez$\mathrm{za}$, un durevole ${ }^{4}$ legame psicologico tra partito ed elettore, modellato dai ricercatori dell'equipe di Campbell analogamente alla relazione tra gruppi sociali e loro membri ${ }^{5}$. È dunque un modello socio-psicologico che vede l'identificazione con il partito come un processo di apprendimento al pari di tutti gli altri ruoli sociali, un processo di interiorizzazione ${ }^{6}$ di fedeltà politiche esterne attraverso sentimenti di appartenenza a gruppi ed identificazioni nell'ambiente sociale.

La party identification, ben lontana dall'essere un prodotto di valutazioni razionali dell'elettore, ricopre al contrario, grazie al mantenimento protratto negli anni delle identità politiche, un ruolo fondamentale nel semplificare il problema della scelta tra le alternative di voto, attraverso la formazione di una sorta di «filtro», uno «schermo percettivo» che seleziona gli elementi di disturbo del proprio sistema di valori politici e garantisce il rafforzarsi del processo di acquisizione di un'identificazione di partito ${ }^{7}$. Una funzione, dunque, strumentale che permette di ri-

${ }^{3}$ In The American Voter (Campbell et al. 1960) si fa riferimento alla letteratura sulla socializzazione politica nella rassegna presentata da Hyman (1959). Relativamente all'applicazione della party identification agli studi sulla socializzazione politica, cfr. Easton e Dennis (1969), Easton e Hess $(1961 ; 1962)$ e Langton (1968). Vedi anche Goldberg $(1969,6-7)$, Miller $(1976,23)$ e Jennings e Niemi (1981). Per quanto concerne ulteriori fonti di sviluppo della party identification cfr. Baker $(1978,124)$ ed anche Mishler et al. (1974).

${ }^{4}$ Riguardo alla stabilità della party identification, alla sua capacità di rafforzarsi in funzione del tempo durante il quale l'elettore rimane fedele al suo partito, cfr. Converse $(1969 ; 1976,12-13)$. Vedi anche Butler e Stokes $(1969,59)$ e Markus e Converse (1979, 1059-1061). Una critica al modello è in Fiorina (1977, 614-615).

'Lo stesso studio dei partiti politici diventa parte del più generale studio dei gruppi e la sola differenza tra partito e gruppo è il suo diretto coinvolgimento nel sistema politico. Cfr. Robertson (1976a, 371).

6 Sulle diverse modalità di interiorizzazione della party identification nei bambini e negli adulti, vedi Baker $(1978,123)$.

${ }_{7}$ Cfr. Campbell et al. $(1964,79)$ e Campbell $(1973,144)$. Sul ruolo della party identification nell'organizzazione percettiva e la strutturazione delle opinioni e gli atteggiamenti su problemi e oggetti politici, vedi anche Davis et al. $(1970,432)$. 
durre la grande quantità di informazioni generate dalla complessità del mondo politico, informazioni difficilmente decodificate dal comune cittadino, spesso lontano da quel mondo e poco motivato a parteciparvi attivamente. L'elettore è alla ricerca di decisional shortcuts (Shively 1979, 1041), di «scorciatoie» attraverso cui raggiungere una decisione nella massa confusa di informazioni politiche ${ }^{8}$.

L'impiego della party identification in molte analisi elettorali, ha comunque dato luogo a interpretazioni non sempre omogenee e non sempre compatibili con la formulazione originaria, sia a causa di problemi dovuti alla procedura di rilevazione impiegata, sia in relazione a più sostanziali problemi di significato'.

Tema fondamentale, negli studi sull'identificazione dell'elettore, è il percorso che conduce all'autocollocazione dell'intervistato sulla dimensione dell'identificazione di partito, come processo di attribuzione di significato le cui tracce svaniscono nelle etichette precostituite della tradizionale scala a sette punti ${ }^{10}$. Il

${ }^{8}$ In The American Voter il partito viene appunto configurato come una «opinion forming agency» (Campbell et al. 1964, 73).

Per questa interpretazione della party identification come mezzo per economizzare l'informazione, cfr. inoltre Butler e Stokes $(1969,60)$, Goldberg $(1969,21)$, Shively $(1972 ; 1979,1041)$. Un'analoga funzione, assolta dal partito, nel connettere l'elettore con il mondo dei grandi problemi politici, di cui spesso al cittadino mancano informazioni sufficienti per strutturare un'opinione precisa, è presente in Spreafico $(1963,726)$. Anche Sani $(1975,542)$, inoltre, ha sottolineato la rilevanza della struttura dei canali di comunicazione e del contenuto dei messaggi politici che raggiungono il pubblico, oltre ai meccanismi di socializzazione, per la formazione e il rafforzamento delle identificazioni partitiche.

Bisogna notare inoltre come la nozione di interesse venga esclusa dal processo di sviluppo della identificazione di partito e così il calcolo razionale delle utilità connesse all'affiliazione con un partito. Sull'argomento cfr. Pizzorno $(1983,14)$.

9 Sono stati avanzati, infatti, dubbi sul suo status di variabile indipendente rispetto alla scelta di voto (Rossi 1959; Thomassen 1976, 69) e sulla sua effettiva antecedenza teorica e temporale, dando luogo ad esiti che in alcuni casi hanno privato il concetto di party identification di caratteristiche essenziali per la sua riconoscibilità. A questo proposito cfr. Fiorina $(1977$; 1978) e Robertson (1976a; 1976b, cap. 6).

${ }^{10}$ Ricordiamo che la metodologia utilizzata dai ricercatori dell'equipe di Campbell, dovendo rilevare una propensione psicologica verso un partito, si basa su di un'autoclassificazione. La domanda originale rivolta agli intervistati è la seguente (Campbell et al. 1954, 90-91): «Generally speaking, do you usually think of yourself as a Republican, a Democrat, an Indipendent, or what?»

(if Republican or Democrat) «Would you call yourself a strong (Republican)

(Democrat) or a not very strong (Republican) (Democrat)?»

(if Indipendent) «Would you call yourself as a closer to the Republican or

Democratic party?»

Ne consegue una scala a sette punti ovvero: Strong Democrat, Weak Democrat, In- 
significato della party identification, il termine di confronto della propria identificazione, il carattere positivo o negativo di questo confronto sono momenti essenziali di tale processo. Occorre cioè comprendere quale sia l'oggetto reale verso cui viene a strutturarsi un sentimento di identificazione; il partito infatti non rappresenta necessariamente il reale e diretto referente delle identificazioni degli elettori, al contrario spesso generate da motivazioni estremamente varie ${ }^{11}$.

Il problema dei significati della party identification ha poi implicazioni che coinvolgono la procedura di rilevazione del concetto $^{12}$, difficoltà che diventano più acute quando la domanda classica deve essere trasferita, «tradotta» in un contesto linguistico e culturale diverso, dove chiedere se ci si «identifica», si è «aderenti» o «sostenitori» di un partito può sottendere significati molto diversi ${ }^{13}$.

dipendent Democrat, Indipendent, Indipendent Republican, Weak Republican, Strong Republican.

1 A questo proposito è opportuno ricordare il contributo di Maggiotto e Pierelson (1977), autori che hanno evidenziato l'esistenza di un'identificazione negativa, un atteggiamento di ostilità verso il partito antagonista. Cfr. inoltre Crewe (1976, 52-53), Feldman e Zuckerman $(1982,211)$ e Field e Andersen $(1969,395)$, ma anche Campbell et al. $(1960,121)$. Altre modalità dell'identificazione negativa vengono definite da Crewe $(1976,52-54)$ che ha inoltre costruito una tipologia delle possibili dimensioni che la party identification può venire ad assumere. Vedi anche la suddivisione di Robertson $(1976 \mathrm{a}, 373)$ tra elettori «know-nothing» ed elettori al contrario ben informati ma comunque identificati.

Crewe $(1976,50-51)$ ha poi sottolineato la rilevanza delle alternative politiche effettivamente disponibili nel sistema politico, nel modellare $\mathrm{i}$ diversi significati che l'identificazione di un individuo può assumere e così la stretta dipendenza della stabilità dell'identificazione dell'elettorato dalle condizioni generali che determinano il mantenimento o meno di una data struttura del sistema politico. Sul tema del rapporto tra stabilità sociale e stabilità della party identification cfr. Converse $(1969 ; 1962)$, Campbell et al. (1960, 280-290; 1966, 136 e ss.) e Campbell (1973, 146-148). Su stabilità delle preferenze di partito e stabilità e omogeneità sociale e politica, vedi poi Berelson et al. (1954, cap. 6).

12 Relativamente alla pluralità dei significati coinvolti dalla domanda classica che traduce operativamente la party identification, cfr. Feldman e Zuckerman $(1982,211)$. Crewe $(1976,53)$ inoltre nota che l'idea di attaccamento psicologico verso un partito viene facilmente confusa con la semplice fedeltà nelle diverse scadenze elettorali, fraintendimento facilitato dalla presenza dei nomi dei partiti nella formulazione tradizionale della domanda. Critiche alla presunta unidimensionalità della party identification sono poi in Weisberg (1980), mentre Miller e Wettenberg (1983) evidenziano ulteriori problemi nella rilevazione degli elettori che non hanno preferenze politiche.

${ }^{13}$ E significativo che, già in un contesto che non presenta difficoltà linguistiche, Butler e Stokes (1969) utilizzino, come hanno notato Crewe (1976) e Robertson (1976a, 366), piuttosto ambiguamente termini quali «partisan self-image», «partisanship», «party allegiance», «party attachment», «party support». E così Barnes $(1984,211)$ rileva che due misure della forza dell'identificazione quali «very close» e «very involved» fanno ri- 
Innanzi tutto appaiono evidenti i limiti della formulazione originaria della domanda, qualora debba essere utilizzata in un diverso contesto politico; questa domanda delimita infatti uno spazio politico continuo definito alle estremità dai due partiti Repubblicano e Democratico ${ }^{14}$, modello non riscontrabile in Europa, dove la presenza di un numero elevato di partiti rende impossibile una definizione univoca delle posizioni dei singoli elettori rispetto al complesso dei partiti politici ${ }^{15}$.

$\mathrm{Al}$ di là dei non indifferenti problemi metodologici, la party identification, anche come mero concetto astratto, è spesso apparsa, fuori dagli Stati Uniti, di dubbia utilità ${ }^{16}$. Altri, cioè, sembrano essere i riferimenti delle identificazioni dell'elettorato europeo; i numerosi cleavages, le «fratture» che segmentano la struttura sociale e che in Italia hanno assunto soprattutto la connotazione delle appartenenze subculturali, costituiscono in realtà i principali meccanismi di canalizzazione degli elettori verso $\mathrm{i}$ partiti ${ }^{17}$. Si tratta di elementi tradizionalmente presenti

ferimento a dimensioni diverse, la prima ad un legame di tipo psicologico, mentre l'altra avrebbe come referente il piano del comportamento, dell'attivismo politico.

${ }^{14}$ Relativamente all'interpretazione spaziale della party identification cfr. Budge et al. (1976, 13-17 e parte II).

is Inoltre su un piano prettamente metodologico, mentre la presenza di due soli partiti negli Stati Uniti permette al ricercatore di specificare chiaramente in un'unica domanda tutte le alternative possibili e all'elettore di mettere a confronto i due partiti, nei sistemi multipartitici europei, l'esistenza di un elevato numero di formazioni politiche, costringe a scegliere se sottoporre all'intervistato l'intera lista dei partiti, elencare solo i partiti più importanti oppure non menzionarne nessuno, con l'effetto di ottenere risultati tutt'altro che simili. Sui diversi esiti di queste scelte cfr. Kaase $(1976,86-87)$ e Crewe $(1989,70-73)$. Relativamente alla maggiore efficacia della party identification in un sistema bipartitico, cfr. Miller $(1976,26)$, Campbell e Valen (1961) e Schleth e Weede (1971).

${ }^{16}$ Ed è proprio l'ottima capacità predittiva dell'identificazione di partito, riguardo al comportamento elettorale nel contesto europeo, ad alimentare i dubbi sulla sua esistenza, in quanto in realtà in Europa gli elettori che cambiano voto cambiano solitamente anche la propria identificazione. Cfr. Budge et al. (1976) e Feldman e Zuckerman $(1982,219)$.

Problemi relativi a cedimenti nell'intensità, nella forza e nella stabilità dell'identificazione dell'elettorato negli Stati Uniti e in Europa, sono stati messi in luce da Dennis (1975), Pomper (1978, cap. 2), Crewe et al. (1977), Feldman e Zuckerman (1982), Niemi et al. (1980), Katz et al. (1980). Sui fenomeni di ridefinizione delle appartenenze politiche, cfr. inoltre Cameron (1972), Inglehart e Hochstein (1972) e Burnham (1970). Relativamente al rapporto tra identificazione di partito ed elettori mobili vedi, invece, Dobson e St. Angelo (1975).

${ }^{17} \mathrm{E}$ così, «in America la party identification fornisce il nesso in base a cui le identificazioni sociologiche hanno un'influenza durevole sulla scelta elettorale» (Butler e Stokes 1969,42$)$. 
nel tessuto sociale, che definiscono in prima istanza le appartenenze dei cittadini e che mediano, attraverso i rapporti preferenziali che i partiti intrattengono con le realtà sociali definite dai diversi cleavages, $\mathrm{i}$ legami tra elettori e partiti ${ }^{18}$.

\section{Un indice additivo di identificazione di partito}

L'esigenza di definire un ambito di significati che siano riconoscibili all'interno della definizione di party identification data dal Michigan Group, impiegando indicatori che possano ricostruire questa accezione di identificazione senza definirla, al contrario, a posteriori, ci porta a sondare l'esistenza, tra l'elettorato italiano ${ }^{19}$, dell'identificazione di partito attraverso un tipo di rilevazione del tutto indiretta di questo fenomeno, mirando a scomporlo in diverse componenti che siano in grado di ricostruirlo nella sua complessità.

A questo scopo, nel presente articolo si utilizzeranno dati provenienti da un sondaggio del $1985^{20}$. Si tratta di dati non certo nuovi, soprattutto alla luce dei grandi mutamenti avvenuti sul piano politico ed elettorale dopo il 1989; essi sono stati comunque utilizzati per via del particolare interesse delle domande presenti, relative sia alle motivazioni di voto dei cittadini, sia alle modalità del loro legame con il partito votato, domande che difficilmente si possono trovare in altre ricerche. Anche se,

18 Sul rapporto tra cleavage e party identification in Europa, vedi anche: Thomassen $(1976,78)$, Shively $(1972,1224 ; 1979,1050 ; 1980,226)$, Le Duc et al. $(1984,483)$, Kaase $(1976,88$ e 101), Baker $(1978,122)$.

19 Il ruolo dell'identificazione di partito negli studi sul comportamento elettorale in Italia è stato finora piuttosto limitato; l'estrema rilevanza, infatti, delle fratture subculturali che ha caratterizzato il nostro Paese e la conseguente impostazione metodologica, che diviene dominante alla fine degli anni sessanta negli studi italiani, basata su di un approccio ecologico e sulla scomposizione del territorio nazionale in zone geo-politiche, e così l'attenzione riposta prevalentemente sul «risultato» e non sul «comportamento» degli elettori (Biorcio e Diamanti 1987, 43), spiega la quasi totale assenza, per lungo tempo, dell'identificazione di partito nelle ricerche italiane. Presenze di questa variabile, utilizzata secondo metodologie diverse, si possono comunque trovare in: Spreafico (1963), Barnes (1971; 1984), Barnes e Pierce (1971), Biorcio e Mannheimer (1986), Mannheimer (1986; 1989), Mannheimer e Sani (1987). Una misura dell'identificazione di partito è anche presente nei sondaggi dell'Eurobarometro. Cfr. inoltre Sani (1975).

${ }_{20} \mathrm{Ci}$ riferiamo alla ricerca «La cultura politica nel Sud Europa», svolta nella primavera del 1985 in Italia, Spagna, Portogallo e Grecia e diretta da Giacomo Sani e Julian Santamaria. Per l'Italia la ricerca è stata condotta da Giovanna Guidorossi, Renato Mannheimer, Leonardo Morlino, Giacomo Sani, Giuliano Urbani e Maria Weber. 
probabilmente, un analogo sondaggio evidenzierebbe oggi una situazione ben diversa e, soprattutto, una minore incidenza dell'identificazione di partito, siamo comunque convinti che i meccanismi che sono alla base delle identificazioni dei cittadini italiani con i loro partiti rimangano gli stessi. Inoltre, l'appartenen$z a$ (nelle diverse forme che può assumere) come modalità del rapporto tra elettore e partito, rappresenta un elemento fondamentale della cultura politica italiana, da cui non si può prescindere nemmeno nell'analisi di nuovi fenomeni politici.

Si farà dunque riferimento, in un primo momento, ad una misura dell'identificazione di partito già impiegata da Mannheimer (1989). Si tratta di un indice additivo, costruito distinguendo due diverse modalità di identificazione, ovvero:

1. un rapporto di vicinanza con un partito che consiste in un «rapporto stretto, esclusivo e dichiarato dell'elettore con il "suo" partito, un rapporto che va necessariamente al di là della situazione politica contingente e che l'elettore sente come una componente stabile e importante del proprio universo di giudizi e opinioni politiche» (Mannheimer 1989, 58);

2. un rapporto di tipo emotivo con il partito politico, che concerne la componente «affettiva» dell'identificazione di partito ed è rivolto a «rilevare la reazione emotivo-psicologica alle contingenze politiche che coinvolgono il partito, la misura in cui l'individuo "vive" e "partecipa" le vicende del suo partito" (Mannheimer 1989, 58).

Le domande poste agli intervistati sono dunque:

1. «Ora le leggerò una lista di partiti. Mi può dire, per favore, se Lei si trova molto vicino, vicino, né vicino né lontano, lontano o molto lontano da ognuno di essi?»

2. «Quando viene criticato il partito per cui Lei ha votato nelle ultime elezioni politiche, di solito che cosa fa, e cioè come reagisce?»

- di solito me la prendo, come se si criticasse anche me;

- di solito non me la prendo, ma mi dispiace;

- di solito resto indifferente, non me la prendo.

Occorre notare che la prima domanda, in apparenza simile per struttura al quesito originario del Michigan Group, se ne discosta fortemente, in quanto qui l'autocollocazione dell'intervistato sulla dimensione vicinanza-lontananza viene richiesta per tutti i partiti e non soltanto per il partito a cui vanno le proprie preferenze; tale formulazione permette di tenere conto del complesso delle dichiarazioni dell'intervistato e valutare 
TAB. 1. Grado di vicinanza al partito

\begin{tabular}{lcc}
\hline Vicinanza al partito & $\%$ & N. \\
molto vicini & 34.1 & 636 \\
vicini & 22.0 & 410 \\
altri & 43.9 & 817 \\
(totale) & $(100.0)$ & $(1863)$ \\
\hline
\end{tabular}

TAB. 2. Grado di coinvolgimento emotivo

\begin{tabular}{lrc}
\hline Reattività emotiva & $\%$ & $\mathrm{~N}$. \\
«me la prendo» & 9.0 & 160 \\
«mi dispiace» & 41.4 & 739 \\
altri & 49.6 & 885 \\
(totale) & $(100.0)$ & $(1784)$ \\
\hline
\end{tabular}

il grado di vicinanza al partito, considerando l'insieme delle distanze che intercorrono tra l'elettore e il complesso delle forze politiche ${ }^{21}$.

La vicinanza e la reattività emotiva ${ }^{22}$ (tabb. 1 e 2 ) sono state quindi composte ${ }^{23}$ in un indice additivo di identificazione di partito, ottenendo così una scala che misura l'identificazione dei cittadini a cinque diversi gradi di intensità (tab. 3 ).

21 È stato infatti considerato «molto vicino» ad un partito chi ha dichiarato di sentirsi «molto vicino» solamente ad un partito, non considerando eventuali altre dichiarazioni di semplice «vicinanza», mentre è stato classificato come «vicino» chi si è dichiarato «vicino» ad un solo partito non dichiarandosi nel contempo «molto vicino» ad alcun partito. Dal campione sono stati poi esclusi coloro che, su un totale di nove partiti, non erano stati capaci di definire il proprio grado di vicinanza nei confronti di sei partiti o più, fatto che pone dei dubbi sulla loro capacità di maneggiare questa dimensione.

Per quanto concerne invece la seconda dimensione, vengono prese in considerazione entrambe le risposte «me la prendo» e «mi dispiace», come parte di un complessivo sentimento di identificazione di partito, a due diversi gradi di intensità.

22 Come nota Mannheimer, sia gli atteggiamenti di vicinanza che quelli di reattività emotiva sono presenti in circa la metà del campione, ma mentre la vicinanza è diffusa a livelli relativamente più forti, il coinvolgimento emotivo viene generalmente espresso attraverso livelli medi di intensità; distribuzione dissimile che «può essere ricondotta alla diversa natura dei due atteggiamenti, ma anche al diverso significato simbolico delle due espressioni con cui li abbiamo misuratis (Mannheimer 1989, 60).

${ }^{23}$ L'opportunità di distinguere queste due dimensioni, la loro rilevanza, è naturalmente subordinata al loro non sovrapporsi, alla capacità di identificare fenomeni separati, non solo a livello teorico, ma anche nell'analisi empirica. Abbiamo, infatti, verificato come le due dimensioni non siano per nulla coincidenti, e, pur mostrando un significativo grado di correlazione, appaiano presenti nell'elettorato anche con segno opposto o comunque a diversi gradi di intensità. Vedi anche Mannheimer $(1989,61)$. 
TAB. 3. Distribuzione degli elettori secondo il grado di intensità dell'identificazione di partito

\begin{tabular}{lcc}
\hline Identificazione di partito & $\%$ & $\mathrm{~N}$. \\
& & \\
indifferenti & 25.4 & 452 \\
neutrali & 27.7 & 495 \\
poco identificati & 23.1 & 412 \\
molto identificati & 18.5 & 330 \\
totalmente identificati & 5.3 & 95 \\
(totale) & $(100.0)$ & $(1784)$ \\
\hline
\end{tabular}

Di fronte ad una comprensibile maggioranza (circa il 50\%) di intervistati che mostrano di essere lontani da un coinvolgimento emotivo o ideale con il partito, il $23 \%$ circa è partecipe di una moderata identificazione, mentre circa il $24 \%$ ( $i$ «molto» e i «totalmente identificati») ha una solida identificazione con un partito. $\grave{E}$ dunque, l'identificazione, una modalità del rapporto con il mondo politico che rimaneva estranea alla metà dell'elettorato del 1985, ma che deve comunque considerarsi un importante elemento per la comprensione del comportamento politico di quasi un quarto del campione.

Si è già discusso delle difficoltà dell'uso dell'identificazione di partito, relativamente alla pluralità delle caratteristiche che questo concetto viene ad assumere nelle diverse ricerche; si cercherà, dunque, di giungere a una più precisa definizione dei suoi contorni e delle sue caratteristiche, tentando di circoscrivere un'area del comportamento di voto, e delle motivazioni che a questo sono collegate, più efficacemente individuabile.

Si è prestato attenzione alle relazioni che intercorrono tra l'identificazione di partito e alcune variabili volte a caratterizzare l'identità politica e sociale dell'elettore.

Abbiamo, innanzi tutto, considerato, oltre al titolo di studio dell'intervistato, le variabili relative al rapporto tra l'elettore e la politica, cioè quegli atteggiamenti e orientamenti di interesse, coinvolgimento e conoscenza, che forniscono una misura del grado di consapevolezza che il cittadino possiede nel muoversi tra gli elementi della politica e così della sua capacità di mettere in atto, in questo ambito, repertori di azione più o meno complessi ed adeguati alle particolari situazioni. In altri termini, si tratta della coscienza dell'elettore di avere spazi autonomi di movimento, oppure del suo affidarsi ad elementi esterni per elaborare le proprie scelte. Inoltre, abbiamo utilizzato un indi- 
TAB. 4. Intensità dell'identificazione di partito in diversi gruppi di intervistati. Relazioni significative (valori percentuali)

\begin{tabular}{|c|c|c|c|c|}
\hline & $\begin{array}{c}\text { poco } \\
\text { identificati }\end{array}$ & $\begin{array}{l}\text { molto } \\
\text { identificati }\end{array}$ & $\begin{array}{l}\text { totalmente } \\
\text { identificati }\end{array}$ & $\begin{array}{l}\text { tutto il } \\
\text { campione }\end{array}$ \\
\hline \multicolumn{5}{|c|}{ Interesse per la politica } \\
\hline basso & 66.7 & 49.4 & 23.4 & 63.8 \\
\hline medio & 28.7 & 41.8 & 43.6 & 29.8 \\
\hline \multirow[t]{3}{*}{ alto } & 4.6 & 8.8 & 33.0 & 6.4 \\
\hline & $(100.0)$ & $(100.0)$ & $(100.0)$ & $(100.0)$ \\
\hline & $(\mathrm{N} .=408)$ & $(\mathrm{N} .=328)$ & $(\mathrm{N} .=94)$ & $\left(N_{.}=1765\right)$ \\
\hline \multicolumn{5}{|c|}{ Partecipazione politica } \\
\hline bassa & 73.8 & 60.1 & 33.7 & 72.8 \\
\hline media & 22.0 & 30.0 & 31.6 & 21.4 \\
\hline \multirow[t]{3}{*}{ alta } & 4.2 & 9.9 & 34.7 & 5.8 \\
\hline & $(100.0)$ & $(100.0)$ & $(100.0)$ & $(100.0)$ \\
\hline & $(\mathrm{N} .=404)$ & $(N .=323)$ & $(\mathrm{N} .=95)$ & $(\mathrm{N} .=1753)$ \\
\hline \multicolumn{5}{|c|}{ Valutazione positiva del partito } \\
\hline bassa & 34.6 & 29.7 & 24.4 & 34.7 \\
\hline media & 39.6 & 34.4 & 31.4 & 37.8 \\
\hline \multirow[t]{3}{*}{ alta } & 25.8 & 35.9 & 44.2 & 27.5 \\
\hline & $(100.0)$ & $(100.0)$ & $(100.0)$ & $(100.0)$ \\
\hline & $(\mathrm{N} .=353)$ & $(N .=306)$ & $\left(N_{.}=86\right)$ & $(\mathrm{N} .=1554)$ \\
\hline
\end{tabular}

catore dell'importanza rivestita dai partiti per l'elettore, allo scopo di fornire una misura del peso assunto dal partito nella formazione della decisione, della sua rilevanza cioè come oggetto fondamentale del voto ${ }^{24}$.

Infine, si è prestato attenzione a come il cittadino percepisce la complessità del mondo politico e, di conseguenza, alla disinvoltura con cui si muove al suo interno. Quello che si desidera comprendere è in quale misura l'elettore sia capace di strutturare autonomamente le proprie opinioni, i propri atteggiamenti su problemi e oggetti politici. In questo senso la domanda impiegata ${ }^{25}$ può dare un'idea della probabilità che l'elettore non si avvicini ad un partito tramite la consapevolezza di

${ }^{24}$ A questo scopo le domande utilizzate sono: «La gente ha opinioni molto diverse sui partiti politici. Vorrei sapere se Lei si trova d'accordo o in disaccordo (molto o abbastanza) con le opinioni che Le leggerò: 1) grazie ai partiti la gente può partecipare alla vita politica dell'Italia; 2 ) senza partiti non ci può essere democrazia».

${ }^{25}$ La domanda è: «Lei si trova d'accordo o in disaccordo con le seguenti opinioni? Molto o abbastanza (d'accordo/in disaccordo)?... la politica è così complicata che spesso la gente come me non può capire quello che veramente succede». 
un'identità tra le proprie valutazioni politiche e le scelte programmatiche del partito, ma che al contrario trovi in quest'ultimo lo strumento più adeguato a guidarlo nello strutturare l'insieme delle proprie opinioni politiche. La domanda del questionario, malgrado non possa da sola provare l'esistenza di un ruolo privilegiato del partito nel guidare $\mathrm{i}$ processi cognitivi dell'elettore, è comunque in grado di circoscrivere un'area del rapporto dell'individuo con la politica e dunque con il partito, in cui lo spazio per un voto che si può caratterizzare come «voto di opinione» appare limitato.

La tab. 4 riporta le variabili che mostrano una relazione significativa con l'identificazione di partito. Il crescente livello di identificazione si accompagna, così, ad un più alto grado di partecipazione politica e di interesse per la politica; ed inoltre chi è identificato si pone di fronte al partito più positivamente di chi ha con esso soltanto uno scarso coinvolgimento, ritenendolo un elemento essenziale per la vita democratica del Paese. Relativamente a quest'ultimo aspetto, non ci è concesso di stabilire l'esistenza della stessa relazione evidenziata da Spreafico $(1963,699)$ tra l'identificazione di partito e la maggiore rilevanza per l'elettore del ruolo del partito rispetto a quello del singolo candidato. La contrapposizione «partito-candidato» è, infatti, un elemento molto importante nel modello di Campbell e della sua équipe, in quanto immediatamente traducibile nell'antinomia tra fattori di lungo e di breve periodo, tra party identification da una parte e short term orientations dall'altra ${ }^{26}$. Le domande disponibili nel nostro questionario purtroppo non consentono una valutazione dell'importanza relativa del partito rispetto al candidato, ma riguardano, più genericamente, il rapporto tra partito e vita democratica del paese e del cittadino; si tratta di variabili che comunque ci permettono di supporre l'esistenza di un percorso privilegiato che lega il cittadino identificato con la propria forza politica, riducendo almeno in parte l'eventuale rilevanza di un rapporto «elettore-candidato» come principale motore della scelta elettorale.

Più interessanti e meno scontati, sono i risultati presentati

26 Si tratta dei due poli attorno ai quali viene spiegato il comportamento di voto nel modello del Michigan Group. Le due più importanti variabili di breve periodo considerate, erano appunto candidates e issues orientations. Cfr. Campbell et al. $(1954,86)$. Critiche alla indipendenza della party identification da variabili di breve periodo sono in Feldman e Zuckerman (1982), Page e Jones (1979) e Markus e Converse (1979). 
TAB. 5. Intensità dell'identificazione di partito in diversi gruppi di intervistati. Relazioni non significative (valori percentuali)

\begin{tabular}{lrrrr}
\hline & $\begin{array}{c}\text { poco } \\
\text { identificati }\end{array}$ & $\begin{array}{c}\text { molto } \\
\text { identificati }\end{array}$ & $\begin{array}{r}\text { totalmente } \\
\text { identificati }\end{array}$ & $\begin{array}{r}\text { tutto il } \\
\text { campione }\end{array}$ \\
$\begin{array}{l}\text { Livello di istruzione } \\
\text { senza titolo }\end{array}$ & 14.8 & 15.8 & 9.5 & 13.8 \\
$\quad$ licenza elem. & 31.8 & 32.1 & 25.3 & 30.2 \\
lic. media inf. & 25.7 & 23.0 & 23.2 & 23.7 \\
diploma & 17.3 & 18.2 & 25.3 & 20.6 \\
laurea e freq. univ. & 10.4 & 10.9 & 16.7 & 11.7 \\
& $(100.0)$ & $(100.0)$ & $(100.0)$ & $(100.0)$ \\
& $(\mathrm{N} .=412)$ & $(\mathrm{N} .=330)$ & $(\mathrm{N} .=95)$ & $(\mathrm{N} .=1784)$ \\
Informazione & & & & \\
bassa & 39.7 & 37.4 & 34.7 & 37.2 \\
media & 28.5 & 26.1 & 22.1 & 28.5 \\
alta & 31.8 & 36.5 & 43.2 & 34.3 \\
& $(100.0)$ & $(100.0)$ & $(100.0)$ & $(100.0)$ \\
& $(\mathrm{N} .=411)$ & $(\mathrm{N} .=329)$ & $(\mathrm{N} .=95)$ & $(\mathrm{N} .=1781)$ \\
Politica complicata & & & & \\
disaccordo & 21.7 & 23.3 & 33.7 & 21.6 \\
abb. d'acc. & 38.6 & 35.3 & 28.4 & 38.6 \\
molto d'acc. & 39.7 & 41.4 & 37.9 & 39.8 \\
& $(100.0)$ & $(100.0)$ & $(100.0)$ & $(100.0)$ \\
& $(\mathrm{N} .=401)$ & $(\mathrm{N} .=326)$ & $(\mathrm{N} .=95)$ & $(\mathrm{N} .=1746)$ \\
\hline
\end{tabular}

nella tab. 5. Le variabili appena viste, relative al livello di partecipazione e di interesse per la politica dell'intervistato, non sono chiaramente interpretabili in quanto a direzione della causalità; essere particolarmente interessato e partecipe, infatti, può spesso considerarsi un atteggiamento che segue l'acquisizione di un'identificazione partitica e non un elemento essenziale per la sua nascita.

Diverso è invece il caso del livello di studio e di informazione (del resto strettamente correlati, in quanto condizionato, il secondo, dalla presenza di un adeguato livello di scolarità), variabili che possono in larga misura dirsi antecedenti rispetto all'identificazione di partito. Il dato interessante è che l'elettorato che condivide un sentimento di identificazione non sembra differenziarsi significativamente rispetto al resto del campione in quanto a titolo di studio e livello di informazione ${ }^{27}$.

${ }^{27}$ Risultato dunque ben diverso da quello raggiunto da Biorcio e Mannheimer che affermano che «il possesso di un sufficiente livello culturale ed informativo appare perciò un importante prerequisito per attribuire un significato alla relazione fra sé ed 
Infine, anche per l'ultima variabile riportata nella tab. 5 non abbiamo riscontrato una relazione significativa con l'identificazione di partito. L'elettore identificato non percepisce il mondo politico come particolarmente complesso e estraneo alla propria capacità di comprensione, anche se bisogna segnalare che è piuttosto l'insieme del campione che si pone a livelli piuttosto alti di accordo con questo item; il $40 \%$ circa del campione, e anche degli identificati, è infatti molto d'accordo con l'opinione che la politica sia tanto complicata da non permettere una reale comprensione delle sue dinamiche.

\section{Identificazione di partito e motivazioni di voto: una tipologia}

L'utilità della party identification risiedeva nella possibilità di distinguere una porzione dell'elettorato che, per la scelta del voto, non fa affidamento su un bilancio delle valutazioni dei partiti in competizione, ma utilizza in primo luogo la propria appartenenza, il sentirsi partecipe di una stessa identità, come strumento di guida. Proprio la funzione strumentale dell'identificazione di partito nel processo di decisione del proprio voto, e ancora prima la sua funzione cognitiva nel dirigere i processi conoscitivi che portano alla rappresentazione del complesso degli attori politici, sono requisiti essenziali per circoscrivere questo concetto, per distinguerlo dalla mera scelta del partito da votare e garantirne l'antecedenza temporale e causale.

L'impossibilità di delineare i tratti di un'identificazione che si caratterizzi significativamente per livello di conoscenza e di informazione e per qualità della scelta elettorale, cioè per il grado di consapevolezza o di eterodirezione con cui questa scelta è attuata, pone dei dubbi sulla capacità di questo indice di identificazione di circoscrivere una realtà in grado di mantenere al suo interno una concreta omogeneità di significati. In tal senso, viene alla luce un ritratto dell'elettore estremamente ambiguo; la stessa possibilità di riconoscere un attore sociale coerente con il modello del gruppo di Campbell sembra, in realtà, perdersi.

un'entità in genere lontana dalla sfera della quotidianità quale il partito votato» (Biorcio e Mannheimer 1986, 115). Tale divergenza è con tutta probabilità da imputarsi alla diversità degli indicatori che sono serviti a stimare la presenza e il livello dell'identificazione di partito; Biorcio e Mannheimer utilizzano infatti soltanto il primo degli items che è stato impiegato nella costruzione del nostro indicatore, ovvero soltanto quello relativo alla reattività emotiva nei confronti delle critiche al proprio partito. 
TAв. 6. Identificazione «con delega» ed identificazione «consapevole»

\begin{tabular}{lcc}
\hline & $\%$ & $\mathrm{~N}$. \\
non identificati & 52.9 & 924 \\
identificazione *consapevole» & 28.2 & 492 \\
identificazione *con delega» & 18.9 & 330 \\
(totale) & $(100.0)$ & $(1746)$ \\
\hline
\end{tabular}

Abbiamo, quindi, preferito distinguere, dall'insieme degli identificati, chi ritiene la politica troppo complicata, ovvero chi mostra di riferirsi, con tutta probabilità, a logiche di scelta di voto che trascendono la valutazione razionale degli elementi del mercato politico ${ }^{28}$. Tale accezione di identificazione ci è parsa più fedele al concetto di party identification, definendo un rapporto di delega verso la propria appartenenza (e verso il partito che la rappresenta) dell'orientamento politico dell'elettore. A questo gruppo di intervistati, che rappresenta circa il 19\% del campione, abbiamo contrapposto il $28 \%$ di coloro che, al contrario, sono consapevoli delle proprie scelte politiche e del legame con il proprio partito (tab. 6). Come si vede, non si è più prestato attenzione alla rilevazione dell'intensità delle identificazioni, ma si è voluto piuttosto focalizzare l'attenzione sulla diversa qualità delle logiche di azione che orientano il comportamento politico dei due gruppi di identificati.

Per la verifica dell'opportunità di tale distinzione, si sono utilizzati degli indici delle motivazioni individuali che muovono la scelta di voto e si è valutato in che misura l'identificazione di partito, così rilevata, sia in grado di circoscrivere aree di motivazioni più coerenti e omogenee.

Abbiamo sottoposto ad analisi fattoriale un elenco di items rappresentanti alcune possibili motivazioni di voto. In questo modo è stato possibile evidenziare tre aree di significato omogeneo, illustrate nella tab. $7^{29}$.

${ }^{28}$ Per la costruzione dell'indice, si è proceduto semplicemente alla suddivisione di tutti gli intervistati che risultavano identificati a qualsiasi livello di intensità, in due sottogruppi in base alle risposte alla domanda, vista in precedenza (nota 25), relativa alla complessità della politica. Quest'ultimo item è stato dicotomizzato separando, da un lato, chi si trovava in disaccordo e chi era abbastanza d'accordo (60\% circa) e, dall'altro, chi era molto d'accordo ( $40 \%)$.

${ }^{29}$ Le dieci motivazioni di voto, sottoposte agli intervistati, sono le seguenti: (1) lo voto solitamente; (2) è votato dai familiari; (3) difende meglio i miei personali interessi e quelli della mia famiglia; (4) è più vicino ai miei ideali; $(5)$ ha uomini più degni di fi- 
TAB. 7. Le tre aree delle motivazioni di voto

\begin{tabular}{|c|c|c|}
\hline $\begin{array}{c}1 \\
\text { Motivazioni ideali- } \\
\text { programmatiche } \\
\text { Orientamento verso } \\
\text { interessi collettivi }\end{array}$ & $\begin{array}{c}2 \\
\text { Interessi immediati } \\
\text { e particolaristici } \\
\text { Relazioni personali }\end{array}$ & $\begin{array}{c}3 \\
\text { Rapporto tradizionale, } \\
\text { consuetudinario } \\
\text { Legame di appartenenza }\end{array}$ \\
\hline $\begin{array}{l}1 . \\
\text { può risolvere i problemi } \\
\text { dell'Italia } \\
2 \text {. } \\
\text { ha uomini più degni di } \\
\text { fiducia } \\
3 \text {. } \\
\text { è più vicino ai miei ideali }\end{array}$ & $\begin{array}{l}1 . \\
\text { mi ha aiutato quando ne ho } \\
\text { avuto bisogno } \\
2 \text {. } \\
\text { ha esponenti che io conosco } \\
\text { personalmente }\end{array}$ & $\begin{array}{l}\text { è votato dai familiari } \\
2 . \\
\text { lo voto solitamente }\end{array}$ \\
\hline
\end{tabular}

Si tratta di una ripartizione che ricalca la tipologia delle relazioni tra partiti ed elettori di Parisi e Pasquino (1977). Occorre comunque notare ${ }^{30}$ che, secondo questi autori, la rappresentazione delle diverse motivazioni di voto coinvolge l'insieme del rapporto che lega gli elettori ai partiti, riferendosi non semplicemente al motivo che porta il cittadino a votare per un certo partito, ma anche al contesto dell'azione di voto, alla base sociale che lo esprime, ai circuiti di comunicazione e alla stabilità nel tempo del rapporto. La nostra tipologia pertanto interseca solo parzialmente quella di Parisi e Pasquino e coinvolge solamente le motivazioni soggettive di voto.

La prima delle tre aree, ovvero quella delle motivazioni ideali-programmatiche, ha come referente un tipo di elettorato più critico, più preparato a valutare la situazione politica, che vede la competizione elettorale come un'azione finalizzata al raggiungimento di benefici collettivi prima che all'interesse privato, dove quindi nella scelta di voto si combina la ricerca di una

ducia; (6) può risolvere meglio i problemi dell'Italia; (7) difende la mia categoria o il mio gruppo sociale; (8) sembra il meno peggio; (9) $\mathrm{mi}$ ha aiutato quando ne ho avuto bisogno; (10) esprime la mia protesta.

Per l'analisi fattoriale abbiamo impiegato il programma Factor di Spss (Statistical package for the social sciences), utilizzando il metodo di estrazione dei fattori $\mathrm{Pa} 2$ e il procedimento di rotazione Varimax. Naturalmente non tutti gli items hanno trovato posto nei tre fattori, ma sono stati inclusi soltanto quelli con un factor loading sufficientemente elevato.

Per analoghe analisi delle motivazioni di voto degli elettori, cfr. Mannheimer e Sani (1987, 52-59) e Mannheimer (1989, 102-106).

${ }^{30}$ Cfr. Mannheimer $(1989,106)$. 
forza politica che rappresenti gli ideali propri e degli uomini in cui si rispecchi la propria volontà politica. È il prevalere della logica di lungo periodo in cui il calcolo degli interessi in gioco si coniuga con l'enfasi verso problemi di più ampio raggio, sia nei termini delle collettività coinvolte, sia della prospettiva temporale.

Sullo stesso piano della soddisfazione di interessi, ma in un'ottica ben diversa, che privilegia da un lato l'interesse individuale rispetto a quello collettivo e dall'altro una logica di breve periodo (dove l'essenziale è la soddisfazione puntuale dei bisogni dell'elettore o se mai del proprio gruppo sociale), si situa invece la seconda area, quella degli interessi.

Infine, in una prospettiva completamente diversa si pone l'area delle motivazioni di appartenenza, rispondente a logiche che rimangono del tutto estranee al calcolo di interesse, qualunque sia la natura collettiva o individuale degli interessi coinvolti. Questo fattore definisce un tipo di scelta che si attua senza tenere conto delle attività e dei programmi dei partiti, ma che fa affidamento sui legami di identità e appartenenza che l'elettore condivide con i familiari o con un più vasto gruppo sociale ad essi connesso, legami consolidati e durevoli che il corso degli avvenimenti politici non mette normalmente in discussione, ma che anzi il rinnovarsi sempre identico della scelta di voto riconferma e ritualizza ${ }^{31}$.

Il primo dato che emerge in relazione alla diversa incidenza delle motivazioni di voto rispetto alla tipologia di identificazione, è la tendenza di tutti e tre i fattori a porsi sempre a livelli superiori rispetto all'intero campione, evidentemente una maggiore capacità degli elettori identificati di razionalizzare le motivazioni delle proprie scelte ${ }^{32}$ (tab. 8 ). La prima area di motiva-

31 Operativamente $\mathrm{i}$ tre fattori sono stati tutti dicotomizzati cercando, in questo modo, di ottenere il maggior grado possibile di omogeneità nelle loro distribuzioni. La capacità discriminante dei diversi items e così dei tre fattori risulta infatti molto diversa; in particolare la prima area, quella delle motivazioni ideali-programmatiche, registra una elevatissima coincidenza (il $68 \%$ circa) delle risposte affermative per tutti e tre gli items.

32 Anche la disponibilità a motivare il proprio voto, a valutare i motivi che portano alla scelta di un partito, misurata attraverso la ricorrenza dei «non so» e dei «non risponde» in risposta agli items sulle motivazioni di voto, è superiore tra gli elettori identificati e lo stesso accade per il numero delle motivazioni fornite, la frequenza delle risposte positive.

Sembra quasi che gli elettori identificati abbiano più motivazioni degli altri per andare alle urne. Certo l'elettore identificato presta una maggiore attenzione all'insieme 
TAB. 8. Identificazione «con delega», identificazione «consapevole» e motivazioni di voto

\begin{tabular}{lcccccc}
\hline & \multicolumn{2}{c}{$\begin{array}{c}\text { motivazioni ideali- } \\
\text { programmatiche }\end{array}$} & $\begin{array}{c}\text { interessi } \\
\text { personali }\end{array}$ & \multicolumn{3}{c}{$\begin{array}{c}3 \\
\text { legame di } \\
\text { appartenenza }\end{array}$} \\
& $\%$ & $\mathrm{~N}$. & $\%$ & $\mathrm{~N}$. & $\%$ & $\mathrm{~N}$. \\
& 79.3 & $(1197)$ & 44.8 & $(713)$ & 56.6 & $(911)$ \\
tutto il campione & & & & & & \\
identificazione «consapevole» & 83.7 & $(380)$ & 49.0 & $(223)$ & 57.4 & $(272)$ \\
identificazione «con delega» & 83.7 & $(251)$ & 46.6 & $(145)$ & 68.7 & $(211)$ \\
\hline
\end{tabular}

zioni, quelle ideali-programmatiche, non presenta elementi di particolare rilievo. E infatti ugualmente diffusa (circa l' $84 \%$ ) in entrambi i tipi di identificazione, ma in realtà gli items alla base di questo fattore riscuotono un'approvazione troppo elevata nell'intero campione per essere in grado di discriminare una porzione dell'elettorato realmente significativa. L'area di motivazioni relativa agli interessi privati vede, invece, una presenza leggermente superiore di chi ha un'identificazione «consapevole» (il $49 \%$ contro il $46,6 \%$ ), sintomo di un rapporto con il partito in qualche misura meno passivo.

Bein differente è la rilevanza della terza area di motivazioni, relativa ai legami di appartenenza tra il cittadino e il suo gruppo di riferimento, che è presente in modo molto diverso nei due gruppi. Circa il $69 \%$ degli intervistati che caratterizzano la propria identificazione con una rapporto di delega, condivide questo tipo di motivazione della scelta di voto, contro il $57,4 \%$ dell'altro gruppo.

L'inserimento della variabile sulla complessità della politica nell'indice di identificazione di partito, sembra in grado di definire l'elettore anche sul piano delle motivazioni di voto, della stessa qualità della scelta di partito. Nell'identificazione «con delega», viene evidenziato un modello di scelta che, oltre a distinguersi in quanto a carenza di quegli elementi di informazione e di consapevolezza che garantiscono la «razionalità» del-

dei fenomeni politici e lo abbiamo visto riguardo alla partecipazione, all'interesse e all'orientamento verso l'oggetto politico nel suo complesso. E un elettore che ha una maggiore predisposizione a tematizzare questo tipo di oggetto ed anche una più elevata necessità di giustificare delle scelte che vengono prese con maggiore forza $e$ un più completo coinvolgimento e che assumono un significato simbolico complesso, che trascende il mero atto del voto per coinvolgere invece l'intera identità del soggetto. 
l'azione, risulta in stretta relazione con la modalità del rapporto che l'elettore intrattiene con il proprio partito.

Il muoversi con difficoltà tra gli oggetti politici e la presenza di un intenso legame con un partito, pone quest'ultimo in un ruolo fondamentale nel plasmare l'orizzonte cognitivo dell'elettore e richiede una sua forte presenza nella guida delle scelte degli identificati.

\section{Caratteri dell'elettore identificato}

Cercheremo ora di definire più dettagliatamente i profili dei due diversi gruppi di elettori identificati. Diversità e punti di contatto si alternano in modo estremamene significativo.

L'identificazione «con delega» è contraddistinta da un basso livello di informazione (il $47,7 \%$ di questo gruppo non legge «mai» o «quasi mai» un quotidiano) e da uno scarso grado di istruzione (più della metà non ha alcun titolo di studio o ha conseguito soltanto la licenza elementare) ${ }^{33}$. Allo stesso modo, risulta piuttosto scarso l'interesse per la politica, mentre superiore ai livelli medi è la partecipazione politica e l'importanza rivestita dal partito politico per l'intervistato (tabb. 9-10).

Si tratta di caratteristiche conformi a come la party identification è stata definita sin dai primi studi del Michigan Group, dove l'elettore identificato viene descritto come un soggetto piuttosto debole dal punto di vista degli strumenti necessari alla scelta di voto e alla formazione di autonome opinioni politiche. Malgrado ciò, il coinvolgimento con il partito politico, sia in termini emotivi che sul piano dell'azione concreta, risulta poi elevato, sorretto com'è dal sentimento di appartenenza degli elettori.

Anche l'età è un elemento rilevante nell'andamento di questo gruppo di identificati. Nella tab. 10 possiamo vedere che quando cresce l'età aumenta anche la quota di elettori che hanno un'identificazione «con delega». Questo fenomeno ha avuto diversi tipi di interpretazione all'interno del modello della party identification. In The American Voter (Campbell et al. 1960,

${ }^{33}$ Si tratta di un risultato piuttosto scontato, implicito nella costruzione dell'indice, se si tiene conto dell'alto livello di correlazione delle due variabili considerate ora e l'opinione discorde riguardo l'item relativo alla complessità della politica. 
161-165) veniva infatti distinto, da un lato, un effetto generazionale, responsabile prevalentemente della direzione dell'identificazione, cioè un orientamento verso un partito che determinate generazioni avevano acquisito storicamente, e, dall'altro, un progressivo aumento nell'intensità dell'identificazione dovuto ad un fenomeno riconducibile al ciclo di vita ${ }^{34}$.

Converse ha sviluppato diversamente il modello, ipotizzando che l'intensità dell'identificazione sia «una funzione non dell'età in sé, ma piuttosto una funzione del periodo di tempo in cui l'individuo ha provato una generica preferenza per un determinato partito e l'ha ripetutamente votato ${ }^{35}$. Occorre dunque interpretare la correlazione tra età e identificazione non tanto come un mero effetto del processo di maturazione dell'individuo, del suo ciclo di vita appunto, ma come l'effetto cumulativo di un legame che è stato mantenuto costante negli anni. Il dato anagrafico sarebbe pertanto un comodo quanto rozzo indicatore di qualcosa di ben diverso, in termini teorici, cioè il perdurare nel tempo di una relazione. Ed infatti Converse aggiunge che quando il tempo viene mantenuto costante, ci si deve aspettare che la forza del legame con il partito sia negativamente correlata con l'età (Converse 1976, 13).

Per quanto concerne la distribuzione degli identificati all'interno di differenti aree professionali, due soli gruppi sono sovrarappresentati tra gli identificati «con delega»; si tratta dei pensionati, che qui sono il $20 \%$ contro il $14 \%$ dell'intero campione, e delle casalinghe (il $28 \%$ contro il $22 \%$ ). Le percentuali più basse sono invece quelle degli studenti e di chi è in cerca di prima occupazione. Ancora una conferma del ruolo che l'età assume nei confronti dell'identificazione di partito; ai poli estremi della distribuzione di questo gruppo di identificati, si collocano, infatti, condizioni professionali decisamente differenziate per quanto concerne l'età. In secondo luogo, è rilevante considerare la posizione di questi gruppi all'interno della struttura

34 D'altra parte anche diverse ricerche italiane hanno verificato l'esistenza di fenomeni legati al ciclo di vita dell'individuo, alla sua «carriera elettorale» (Biorcio e Natale $1989,397)$, fenomeni che determinano il passaggio da «una fase iniziale di apprendimento, con una maggiore disponibilità alla sperimentazione e alla esplorazione di diverse opzioni di voto», ad una «successiva fase di relativa stabilizzazione delle scelte elettorali durante la quale, in relazione ai diversi livelli di identificazione partitica, si creano maggiori resistenze al cambiamento» (Biorcio e Natale 1989, 397-398). Cfr. anche Mannheimer $(1986,51-55)$.

${ }^{35}$ Cfr. Converse $(1976,12-13)$, traduzione nostra. 
TAв. 9. Identificazione «con delega» e identificazione kconsapevole» secondo alcune caratteristiche sociali e demografiche (valori percentuali)

\begin{tabular}{|c|c|c|c|}
\hline & $\begin{array}{l}\text { identificazione } \\
\text { «consapevole» }\end{array}$ & $\begin{array}{l}\text { identificazione } \\
\text { «con delega» }\end{array}$ & $\begin{array}{l}\text { tutto il } \\
\text { campione }\end{array}$ \\
\hline \multicolumn{4}{|l|}{ Livello di istruzione } \\
\hline senza titolo & 8.3 & 23.0 & 13.5 \\
\hline licenza elem. & 25.4 & 39.4 & 30.2 \\
\hline lic. media inf. & 26.8 & 21.2 & 23.6 \\
\hline diploma & 23.2 & 12.4 & 21.0 \\
\hline \multirow[t]{3}{*}{ laurea e freq. univ. } & 16.3 & 4.0 & 11.7 \\
\hline & $(100.0)$ & $(100.0)$ & $(100.0)$ \\
\hline & $(N .=492)$ & $(\mathrm{N} .=330)$ & $(\mathrm{N} .=1746)$ \\
\hline \multicolumn{4}{|l|}{ Età } \\
\hline $18-24$ & 15.7 & 8.2 & 14.4 \\
\hline $25-34$ & 21.7 & 14.8 & 19.9 \\
\hline $35-44$ & 24.0 & 18.5 & 21.5 \\
\hline $45-54$ & 15.6 & 22.1 & 18.3 \\
\hline 55.64 & 10.8 & 18.2 & 13.5 \\
\hline \multirow[t]{3}{*}{65 e oltre } & 12.2 & 18.2 & 12.4 \\
\hline & $(100.0)$ & $(100.0)$ & $(100.0)$ \\
\hline & $(\mathrm{N} .=492)$ & $(\mathrm{N} .=330)$ & $\left(N_{.}=1746\right)$ \\
\hline \multicolumn{4}{|c|}{ Condizione professionale } \\
\hline Lav. autonomi & 18.5 & 12.4 & 15.7 \\
\hline Dip. set. pub. & 15.8 & 11.8 & 14.6 \\
\hline Dip. set. priv. & 22.0 & 20.9 & 21.1 \\
\hline Disoccupati & 3.3 & 2.7 & 3.4 \\
\hline Pensionati & 12.0 & 20.1 & 14.1 \\
\hline In cerca I occup. & 2.2 & 1.8 & 2.6 \\
\hline Studenti & 8.9 & 2.1 & 6.2 \\
\hline \multirow[t]{3}{*}{ Casalinghe } & 17.3 & 28.2 & 22.3 \\
\hline & $(100.0)$ & $(100.0)$ & $(100.0)$ \\
\hline & $(\mathrm{N} .=492)$ & $(\mathrm{N} .=330)$ & $(\mathrm{N} .=1746)$ \\
\hline
\end{tabular}

sociale: appare evidente la collocazione relativamente marginale degli identificati «con delega», pensionati e casalinghe, confermata poi dall'alta percentuale di coloro che hanno un basso livello di istruzione.

Gli identificati «consapevoli», diversamente dagli altri, hanno un alto interesse per la politica ed anche elevati livelli di informazione e di istruzione; circa il $40 \%$ legge tutti i giorni un quotidiano ed un'analoga quota di intervistati ha almeno il diploma di scuola media superiore. Allo stesso modo degli identificati «con delega», mostrano, invece, una partecipazione politica maggiore rispetto all'insieme del campione (ed in realtà più elevata dell'altro gruppo di identificati) ed una valutazione più 
TAB. 10. Identificazione «con delega», identificazione «consapevole» e atteggiamenti politici degli intervistati (valori percentuali)

\begin{tabular}{|c|c|c|c|}
\hline & $\begin{array}{l}\text { identificazione } \\
\text { «consapevole» }\end{array}$ & $\begin{array}{l}\text { identificazione } \\
\text { «con delega» }\end{array}$ & $\begin{array}{l}\text { tutto il } \\
\text { campione }\end{array}$ \\
\hline $\begin{array}{c}\text { Informazio } \\
\text { bassa } \\
\text { media } \\
\text { alta }\end{array}$ & $\begin{array}{r}31.0 \\
28.1 \\
40.9 \\
(100.0) \\
(\mathrm{N} .=491)\end{array}$ & $\begin{array}{r}47.7 \\
25.8 \\
26.5 \\
(100.0) \\
(\mathrm{N} .=329)\end{array}$ & $\begin{array}{r}36.5 \\
28.8 \\
34.7 \\
(100.0) \\
(\mathrm{N} .=1743)\end{array}$ \\
\hline $\begin{array}{c}\text { Interesse p } \\
\quad \text { basso } \\
\text { medio } \\
\text { alto }\end{array}$ & $\begin{array}{r}45.5 \\
42.4 \\
12.1 \\
(100.0) \\
(\mathrm{N} .=488)\end{array}$ & $\begin{array}{r}67.6 \\
26.6 \\
5.8 \\
(100.0) \\
(\mathrm{N} .=327)\end{array}$ & $\begin{array}{r}63.3 \\
30.2 \\
6.5 \\
(100.0) \\
(\mathrm{N} .=1727)\end{array}$ \\
\hline $\begin{array}{l}\text { Partecipazi } \\
\text { bassa } \\
\text { media } \\
\text { alta }\end{array}$ & $\begin{array}{r}57.3 \\
30.3 \\
12.4 \\
(100.0) \\
(\mathrm{N} .=482)\end{array}$ & $\begin{array}{r}72.0 \\
21.5 \\
6.5 \\
(100.0) \\
(\mathrm{N} .=325)\end{array}$ & $\begin{array}{r}72.4 \\
21.8 \\
5.8 \\
(100.0) \\
(\mathrm{N} .=1716)\end{array}$ \\
\hline $\begin{array}{l}\text { Valutazione } \\
\text { bassa } \\
\text { media } \\
\text { alta }\end{array}$ & $\begin{array}{r}30.2 \\
37.9 \\
31.9 \\
(100.0) \\
(\mathrm{N} .=464)\end{array}$ & $\begin{array}{r}33.3 \\
33.9 \\
32.8 \\
(100.0) \\
(\mathrm{N} .=274)\end{array}$ & $\begin{array}{r}34.7 \\
37.7 \\
27.6 \\
(100.0) \\
(\mathrm{N} .=1538)\end{array}$ \\
\hline
\end{tabular}

positiva della rilevanza del partito politico. L'età non è invece rilevante in questo tipo di identificazione, mentre, specularmente al resto degli identificati, è presente un'elevata quota di studenti (tabb. 9-10).

A fronte di un gruppo di identificati che si rivela ben consapevole della propria appartenenza, coloro che hanno un'identificazione «con delega» sono, al contrario, elettori collocati in ruoli relativamente marginali della struttura sociale, sia rispetto al sistema produttivo e quindi alle garanzie sociali che ne derivano, sia rispetto alle risorse informative e culturali necessarie alla messa in atto di un «voto di opinione». Il voto espresso viene in gran parte determinato dal partito e risulta ben poco legato alla particolare consultazione ed alla congiuntura politica. L'affiliazione partitica rappresenta, in questo caso, uno stru- 
TAB. 11. Identificazione «con delega» e identificazione «consapevole» nell'elettorato dei partiti (valori percentuali)

\begin{tabular}{|c|c|c|c|c|c|}
\hline & $\begin{array}{c}\text { non } \\
\text { identificati }\end{array}$ & $\begin{array}{l}\text { identificazione } \\
\text { «consapevole» }\end{array}$ & $\begin{array}{l}\text { identificazione } \\
\text { «con delega» }\end{array}$ & & \\
\hline & $\%$ & $\%$ & $\%$ & $\%$ & N. \\
\hline \multicolumn{6}{|l|}{ Partiti } \\
\hline Msi & 40.0 & 25.5 & 34.5 & $(100.0)$ & 55 \\
\hline$P c i$ & 34.0 & 37.7 & 28.3 & $(100.0)$ & 353 \\
\hline$D c$ & 40.8 & 34.0 & 25.2 & $(100.0)$ & 441 \\
\hline Psi & 49.5 & 29.3 & 21.2 & $(100.0)$ & 222 \\
\hline$D p-P r$ & 51.2 & 39.0 & 9.8 & $(100.0)$ & 41 \\
\hline Psdi-Pri-Pli & 58.4 & 34.5 & 7.1 & $(100.0)$ & 113 \\
\hline
\end{tabular}

mento attraverso cui l'elettore diviene capace di affermare e poi stabilizzare delle scelte assunte in condizioni di scarsità di informazione e incertezza riguardo alle complesse dinamiche politiche presenti al momento del voto. $\grave{E}$ uno strumento radicato nella specificità di un rapporto tra elettore e partito che privilegia la costruzione e il rafforzamento delle identità dei suoi membri, dove anche la scelta di voto diviene un semplice corollario dei processi di affermazione delle appartenenze.

Utili informazioni offrono anche le variabili più propriamente politiche. Rilevanti differenze nella distribuzione degli identificati sono prodotte dalla preferenza di partito dichiarata dagli intervistati (tab. 11). Il Pci e la Dc hanno, al loro interno, considerevoli quote di elettori identificati, sia dell'uno che dell'altro tipo, mentre l'Msi appare fortemente caratterizzato (per il $34,5 \%$ di coloro che lo scelgono) dalla presenza dell'identificazione «con delega». Al contrario, Democrazia proletaria e Partito radicale, da un lato, e i partiti laici (Pli, Pri e Psdi), dall'altro, presentano considerevoli quote di elettori con un identificazione «consapevole» (rispettivamente con il $39 \%$ e il $34,5 \%$ ).

Evidentemente le differenze esistenti tra i partiti non sono giustificabili semplicemente nei termini del rapporto tra partiti estremisti e partiti moderati o di quello tra partiti d'opposizione e partiti di governo, ma sono da ricondurre alle peculiarità di ogni partito, alla sua specifica cultura politica. In questo senso le alte percentuali di identificati, di entrambi i tipi, trovate nell'elettorato della Dc e del Pci sono da collocare all'interno della presenza nel nostro Paese di forti tradizioni culturali e politiche, dotate di grande capacità di penetrazione nelle strutture 
TAB. 12. Identificazione «con delega», identificazione "consapevole» $e$ autocollocazione politica (valori percentuali)

\begin{tabular}{crrr}
\hline & $\begin{array}{c}\text { identificazione } \\
\text { «consapevole» }\end{array}$ & $\begin{array}{c}\text { identificazione } \\
\text { «con delega» }\end{array}$ & $\begin{array}{c}\text { tutto il } \\
\text { campione }\end{array}$ \\
$\begin{array}{c}\text { Continuum sinistra-destra } \\
\text { Sinistra 1 }\end{array}$ & 18.9 & 20.0 & 12.8 \\
2 & 10.4 & 11.1 & 8.9 \\
3 & 9.8 & 10.7 & 11.4 \\
4 & 9.3 & 7.5 & 10.4 \\
5 & 25.0 & 22.0 & 26.7 \\
6 & 11.5 & 10.4 & 13.0 \\
7 & 4.2 & 3.6 & 5.0 \\
8 & 4.8 & 5.0 & 4.8 \\
9 & 2.2 & 2.9 & 2.1 \\
10 & 3.9 & 6.8 & 4.9 \\
& $(100.0)$ & $(100.0)$ & $(100.0)$ \\
Destra & $(\mathrm{N} .=460)$ & $(\mathrm{N} .=280)$ & $(\mathrm{N} .=1465)$ \\
& & & 4.4 \\
media & 4.4 & 4.3 & 0.514 \\
\hline
\end{tabular}

sociali di base (famiglia, comunità, ecc.) $)^{36}$. Inoltre, la composizione dell'elettorato di entrambi questi partiti - come, in parte, del Movimento sociale - comprende settori numericamente rilevanti di cittadini scarsamente istruiti, poco informati e con un basso interesse per la politica e spiega la presenza delle più alte quote di identificati «con delega». Al contrario i partiti laici, Democrazia proletaria e il Partito radicale, sono forze politiche scelte da elettori dotati di elevato grado di istruzione e informazione politica, meno propensi a costruire un rapporto di delega con il partito.

L'autocollocazione media sull'asse sinistra-destra degli intervistati identificati non è, invece, molto diversa da quella dell'intero campione (tab. 12). La distribuzione risulta, però, più dispersa $^{37}$, con minori frequenze nelle posizioni centrali ed una maggiore rilevanza delle estremità del continuum, specie tra gli identificati «con delega».

${ }^{36}$ Per quanto concerne il rapporto tra identificazione di partito e fedeltà elettorale da una parte e appartenenza subculturale dall'altra, due concetti vicini alla party identification e spesso a questa contrapposti, abbiamo verificato l'indipendenza e la non coincidenza, anche sul piano empirico e non soltanto in base agli assunti teorici, dell'indicatore tipologico di identificazione di partito da queste variabili. Cfr. anche Mannheimer $(1989,63-70)$.

37 A questo proposito, nella tab. 12, oltre alla distribuzione di frequenze, viene riportato anche il coefficiente di variazione $(\mathrm{V})$. 
Come abbiamo visto, malgrado le due modalità di identificazione si pongano per caratteristiche sociali degli intervistati, risorse informative e logiche di azione coinvolte, su piani ben diversi, i comportamenti politici a cui danno luogo presentano rilevanti sovrapposizioni. Simili sono, infatti, i livelli di partecipazione politica e l'atteggiamento relativo alla rilevanza dei partiti, come anche alcuni aspetti della collocazione politica ed una contemporanea presenza nell'elettorato di alcuni partiti.

\section{Due identificazioni}

Le due accezioni di identificazione di partito coinvolgono elettori con caratteristiche estremamente differenti, ma ugualmente fanno riferimento alla dimensione dell'appartenenza come modalità di orientamento tra gli oggetti politici. Ci troviamo di fronte ad identificazioni che, se da una parte coinvolgono motivazioni di voto $\mathrm{e}$, in generale, di agire politico, del tutto dissimili, dall'altra rappresentano entrambe una modalità diretta del rapporto tra elettore e partito, ovvero appartenenze che definiscono la propria identità essenzialmente a partire dal partito politico.

Se da un lato esiste un'identificazione che trova nel rapporto con il partito una risorsa fondamentale per la possibilità di dare senso al proprio agire e alla percezione di sé come attore politico, dall'altro il sentimento di identificazione è posto alla fine di un percorso che, da orientamenti politici già ben strutturati, giunge al partito per riconoscervi una forza capace di dare espressione collettiva alla propria identità così da riconfermarla e protrarla nel tempo.

Un'identificazione dunque strumentale, sostanzialmente riconducibile al modello del Michigan Group, contro un'identificazione, al contrario, consapevole della propria appartenenza e del significato della propria scelta, creata da quegli strumenti che proprio al primo tipo mancano e ne motivano l'esistenza.

La rilevanza anche sul piano del comportamento politico è quindi ovvia: se da un lato l'agire è in gran parte eterodiretto, incurante della contingenza politica, impermeabile a tutto ciò che è esterno alla propria appartenenza, dall'altro l'elettore appare dotato di una elevata capacità di attuare scelte autonome $e$ di affermare attraverso di esse i propri principi politici. Non c'è dubbio che molte somiglianze permangono tra $i$ due tipi di at- 
tori. Entrambi condividono infatti una stabilità nel comportamento di voto che rimane mediamente più elevata rispetto all'elettore non identificato. Anche sul piano della strutturazione delle opinioni e degli atteggiamenti politici, l'essere identificati con un partito può comunque comportare una maggiore conformità verso le opinioni del gruppo di cui si fa parte, rispetto a chi invece non si identifica affatto.

Il dato comunque rilevante è che, almeno all'epoca del sondaggio, l'esistenza di queste diverse modalità di identificazione, rappresentava una realtà probabilmente peculiare del contesto italiano, non riscontrabile in analoghe ricerche europee e tanto meno americane. La forte caratterizzazione ideologica dell'elettorato italiano, la rilevanza e la pervasività sociale dei partiti presenti non solo all'interno del sistema politico, ma anche nell'intera società e dunque capaci di disegnarne i confini, di strutturare il complesso dei diversi contesti sociali - ha reso possibile la formazione di appartenenze politiche tanto diverse nell'identità degli attori coinvolti.

Indubbiamente l'ipotesi avrebbe bisogno di ulteriori verifiche sul piano empirico, attraverso dati comparati, dotati di indicatori adatti non solo a cogliere le possibili dimensioni dell'identificazione di partito, ma anche a caratterizzare l'identità politica degli elettori identificati, con un'appropriata rilevazione delle motivazioni del comportamento di voto e dell'agire politico. È necessario focalizzare l'attenzione sui processi di produzione di senso, sull'interpretazione dell'agire e non tanto sulla sua causa e il suo scopo, e indagare le pratiche di produzione di significato come processi non confinabili unicamente nell'ambito della dimensione privata dell'individuo, ma al contrario da ricondursi all'interno di ben strutturati contesti relazionali ${ }^{38}$.

Occorre inoltre ricordare che il quadro tracciato si riferisce all'Italia del 1985. Gran parte delle ricerche sul comportamento politico indicano un progressivo venir meno delle appartenenze dei cittadini ed anche i più recenti avvenimenti politici mostrano chiaramente che $i$ nuovi equilibri elettorali verranno giocati su piani ben diversi. La comprensione di fenomeni nuovi nel rapporto tra elettori e partiti non potrà comunque evitare il

${ }^{38}$ Cfr. Giglioli (1989, 132-133), Luhmann (1975; trad. it. 1979, 20 e ss.). Sui temi legati all'agire e all'attore sociale vedi anche Gallino (1985), Biorcio e Diamanti (1987) e Pizzorno $(1983 ; 1986 ; 1989)$. 
confronto con i meccanismi del passato e le stesse vecchie appartenenze contribuiranno a plasmare i nuovi rapporti, se non altro opponendo un freno al cambiamento.

L'affievolirsi anche in Italia della capacità identificante dei partiti rende comunque probabile lo sviluppo della party identification nel nostro Paese in direzione simile a quella delle altre nazioni europee, con il venir meno di meccanismi di identificazione da parte di quegli elettori che dispongono di un'elevata autonomia nell'elaborazione dei propri orientamenti politici. In tale contesto, il rapporto di «delega» potrebbe comunque rappresentare un elemento di continuità con il passato, costituendo, pur in una differente situazione, un'importante modalità del rapporto tra cittadini e sistema politico per quella parte dell'elettorato che meno è in grado di sviluppare autonomamente le proprie convinzioni politiche.

\section{Riferimenti bibliografici}

Baker, K. (1978), Generational Differences in the Role of Political Behaviour, in «American Journal of Political Science», XXII, pp. 106-129.

Barnes, S.H. (1971), Modelli spaziali e l'identificazione partitica dell'elettore italiano, in «Rivista Italiana di Scienza Politica», II, pp. 123-143.

- (1984), Secular Trends and Partisan Realignment in Italy, in R.J. Dalton, S.C. Flanagan e P.A. Beck (a cura di), Electoral Change in Advanced Democracies, Princeton, Princeton University Press, pp. 205-230.

Barnes, S.H. e R. Pierce (1971), Public Opinion and Political Preferences in France and Italy, in «Midwest Journal of Political Science», XV, pp. 643-660.

Belknap, G. e A. Campbell (1952), Political Party Identification and Attitudes Toward Foreign Policy, in «Public Opinion Quarterly», XV, pp. 601-623.

Berelson, B., P.F. Lazarsfeld e W. McPhee (1954), Voting, Chicago, University of Chicago Press.

Biorcio, R. e I. Diamanti (1987), La scelta di voto: dal risultato all'attore sociale. Appunti per una rilettura del comportamento elettorale in Italia, in «Quaderni dell'Osservatorio Elettorale», n. 19, pp. 43-86.

Biorcio, R. e R. Mannheimer (1986), Elettorato e fratture culturali, in R. Biorcio e R. Mannheimer, Misurare la Politica, Milano, Unicopli, pp. 113-143, precedentemente in «Politica ed Economia», marzo 1985. 
Biorcio, R. e P. Natale (1989), La mobilità elettorale degli anni ottan$t a$, in «Rivista Italiana di Scienza Politica», XIX, pp. 385-430.

Budge, I., I. Crewe e D. Farlie (a cura di) (1976), Party Identification and Beyond, London, Willey.

Burnham W.D. (1970), Critical Elections and the Mainspring of American Politics, New York, Norton.

Butler, D.E. e D.E. Stokes (1969), Political Change in Britain, London, MacMillan.

Cameron, D.M. (1972), Stability and Change in Patterns of French Partisanship, in «Public Opinion Quarterly», XVI, pp. 19-30.

Campbell, A. (1973), Alla ricerca di un modello in psicologia elettorale comparata, in G. Urbani (a cura di), La politica comparata, Bologna, Il Mulino, pp. 139-158.

Campbell, A., P.E. Converse, W.E. Miller e D.E. Stokes (1960), The American Voter, New York, Willey.

- (1964), The American Voter: an abridgment, New York, Willey.

- (1966), Election and the Political Order, New York, Willey.

Campbell, A., G. Gurin e W.E. Miller (1954), The Voter Decides, Row, Evaston, Peterson \& co.

Campbell, A. e H. Valen (1961), Party Identification in Norway and the United States, in Campbell et al. (1966), pp. 245-268.

Converse, P.E. (1962), Information Flow and the Stability of Partisan Attitudes, in «Public Opinion Quarterly», XXVI, pp. 578-599.

- (1966), The Concept of a Normal Vote, in Campbell et al. (1966), pp. 9-39.

- (1969), Of Time and Partisan Stability, in «Comparative Political Studies», II, pp. 139-171.

- (1976), The Dynamics of Party Support, Beverly Hills, Sage.

Crewe, I. (1976), Party Identification Theory and Political Change in Britain, in Budge et al. (1976), pp. 33-61.

- (1989), I sondaggi elettorali in Gran Bretagna: problemi di disegno della ricerca, risposta e distorsione, in $\mathrm{R}$. Mannheimer (a cura di), I sondaggi elettorali e le scienze politiche. Problemi metodologici, Milano, Franco Angeli, pp. 147-196; inizialmente pubblicato in «Essex Papers in Politics and Government», n. 6, ottobre 1983.

Crewe, I., B. Sarlvick e J. Alt (1977), Partisan Realigments in Britain 1964-1974, in «British Journal of Political Science», VII, pp. 129. 190.

Dennis, J. (1975), Trends in Support for the American Party System, in «British Journal of Political Science», V, pp. 187-230.

Davis, O.A., M.J. Hinich e P.C. Ordescook (1970), An Expository Developement of a Mathematical Model of the Electoral Process, in «American Political Science Review», LXIV, pp. 426-448.

Dobson D. e D. St. Angelo (1975), Party Identification and the Floating Vote: some dynamics, in «American Political Science Review», LXIX, pp. 481-490. 
Easton, D. e J. Dennis (1969), Children in the Political System, New York, Mcraw Hill.

Easton, D. e R.D. Hess (1961), Youth and the Political System, in S.M. Lipset e L. Lowentahal (a cura di), Culture and Social Characters, Glencoe, Free Press.

- (1962), The Child's Political World, in «Midwest Journal of Political Science», VI, n. 3, pp. 229-246.

Feldman, S. e A.S. Zuckerman (1982), Partisan Attitudes and the Vote, in «Comparative Political Studies», XV, n. 2, pp. 197-222.

Field, J.O. e R.E. Anderson (1969), Ideology in the Public's Conceptualization of the 1964 Election, in «Public Opinion Quarterly», XXXIII, pp. 380-398.

Fiorina, M. (1977), An Outline for a Model of Party Choice, in «American Journal of Political Science», XXI, pp. 601-625.

- (1978), Economic Retrospective Voting in America National Election: a micro-analysis, in «American Journal of Political Science», XXII, pp. 426-443.

Gallino, L. (1985), Il mancato sviluppo d'una teoria dell'attore nella sociologia italiana, in «Quaderni di Sociologia», n. 4-5.

Giglioli, P.P. (1989), Teorie dell'azione, in A. Panebianco (a cura di), L'analisi della politica, Bologna, Il Mulino, pp. 107-133.

Goldberg, A.S. (1969), Social Determinism and Rationality as a Basis of Party Identification, in «American Political Science Review», LXIII, pp. 5-25.

Hyman H. (1959), Political Socialization, Glencoe, Illinois, Free Press.

Inglehart, R. e A. Hochstein (1972), Alignment and Dealignment of the Electorate in France and United States, in «Comparative Political Studies», V, pp. 343-372.

Jennings, M.K. e R. Niemi (1981), Generations and Politics, Princeton, Princeton University Press.

Kaase, M. (1976), Party Identification and Voting Bebaviour in the West German Election of 1969, in Budge et al. (1976), pp. 81-102.

Langton, K.P. (1968), Political Socialization, Oxford, Oxford University Press.

Le Duc, L., H.D. Clarke, J. Jenson e J.H. Pammet (1984), Partisan instability in Canada: Evidence from a New Panel Study, in «American Political Science Reviews, LXXIIX, pp. 470-484.

Luhmann, N. (1975), Macht, Stuttgart, Enke, trad. it. Potere e complessità sociale, Milano, Il Saggiatore, 1979.

Maggiotto, M. e J.E. Piereson (1977), Party Identification and Electoral Choice: the Hostility Hypothesis, in «American Journal of Political Science», XXI, pp. 745-768.

Mannheimer, R. (1986), Come spiegare la mobilità elettorale in Italia, in «Rivista Italiana di Scienza Politica», XVI, n. 1, pp. 45-80.

- (1989), Capire il voto, Milano, Franco Angeli. 
Mannheimer, R. e G. Sani (1987), Il mercato elettorale, Bologna, Il Mulino.

Markus, G.B. e P.E. Converse (1979), A Dynamic Simultaneous Equation Model of Electoral Choice, in «American Political Science Review», LXXIII, pp. 1055-1070.

Marradi, A. (1980), Concetti e metodi per la ricerca sociale, Firenze, La Giuntina.

Miller, A. e M. Wettenberg (1983), Measuring Party Identification: Indipendent or No Partisan Preference?, in «American Journal of Political Science», XXVII, pp. 106-121.

Miller, W.E. (1976), The Cross-National Use of Party Identification as a Stimulus to Political Inquiry, in Budge et al. (1976), pp. 21-31.

Mishler, W., A. Kornberg, D. Lindquist e J. Smith (1974), Patterns of Political Socialization: Simulating the Developement of Party Identification in Two Political Elites, in «Comparative Political Studies», VI, pp. 399-430.

Niemi, R., R. Katz e D. Newman (1980), Recostructing Part Partisanship: the Failure of the Party Identification Recall Questions, in «American Journal of Political Science», XXIV, pp. 633-651.

Page, B.I. e C. Jones (1979), Reciprocal Effects of Policy Preferences, Party Loyalties and the Vote, in «American Political Science Review», LXXIII, pp. 1071-1089.

Parisi, A. e G. Pasquino (1977), Relazioni partiti-elettori e tipi di voto, in A. Parisi e G. Pasquino (a cura di), Continuità e mutamento elettorale in Italia, Bologna, Il Mulino, pp. 215-249.

Pizzorno, A. (1983), Sulla razionalità della scelta democratica, in «Stato e Mercato», n. 7, pp. 3-46.

- (1986), Sul confronto intertemporale delle utilità, in «Stato e Mercato», n. 16, pp. 3-25.

- (1989), Spiegazione come reidentificazione, in «Rassegna Italiana di Sociologia», XXX, n. 2, pp. 161-184.

Pomper, G. (1978), Voter's Choice, New York, Dodd Mead.

Prewitt, K. e N.H. Nie (1971), Election Studies of the Survey Research Center, in «British Journal of Political Science», I, pp. 479-502.

Robertson, D. (1976a), Surrogate for Party Identification in the Rational Choice Framework, in Budge et al. (1976), pp. 365-381.

- (1976b), A Theory of Party Competition, London, Willey.

Rossi, P.H. (1959), Four Landmarks in Voting Research, in E. Burdick e A. Brodbeck (a cura di), American Voting Behaviour, New York, Free Press.

Sani, G. (1975), Ricambio elettorale e identificazioni partitiche: verso una egemonia delle sinistre?, in «Rivista Italiana di Scienza Politica», V, n. 3, pp. 515-544.

Schleth, U. e E. Weede (1971), Causal Models on West-German Voting Bebaviour, in R. Wildenmann (a cura di), Sozialwissenschaftliches Jabrbuck für Politik, München, Günter Olzog, pp. 73-98. 
Shively, W.P. (1972), Party Identification, Party Choice and Voting Stability: the Weimar Case, in «American Political Science Review», LXVI, pp. 1203-1225.

- (1979), The Development of Party Identification Among Adults: Exploration of a Functional Model, in «American Political Science Review», LXXIII, pp. 1039-1054.

- (1980), The Nature of Party Identification: a Review of Recent Developments, in J.C. Pierce e J.L. Sullivan (a cura di), The Electorate Reconsidered, Beverly Hills, Sage.

Spreafico, A. (1963), Orientamento politico e identificazione partitica, in A. Spreafico e J. La Palombara (a cura di), Elezioni e comportamento politico in Italia, Milano, Comunità, pp. 687-731.

Thomassen, J. (1976), Party Identification as cross-national concept: its Meaning in the Netherlands, in Budge et al. (1976), pp. 63-79.

Weisberg, H. (1980), A Multidimensional Conceptualization of Party Identification, in «Political Behaviour», n. 2, pp. 33-60. 